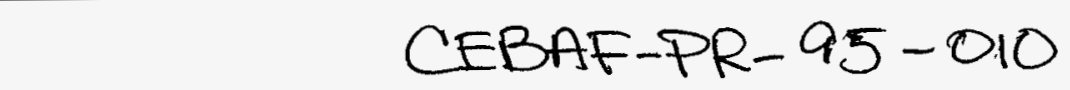

$$
D \circ E / E R / 40 / 50--320
$$

\title{
AUTOMATIC PUMPDOWN OF THE 2K COLD COMPRESSORS FOR THE CEBAF CENTRAL HELIUM LIQUEFIER*
}

\author{
B. S. Bevins, W. C. Chronis, and M. S. Keesee \\ Continuous Electron Beam Accelerator Facility \\ 12000 Jefferson Avenue \\ Newport News, VA 23606-1909 USA
}

\begin{abstract}
The $2 \mathrm{~K}$ system at CEBAF includes four stages of centrifugal cold compressors that operate between a suction pressure of $0.031 \mathrm{~atm}$ and a discharge of $\sim 1 \mathrm{~atm}$. Starting the cold compressors and pumping the system down to operating pressure is a complex and time consuming operation. Many months of rigorous testing were required to develop a reliable startup procedure. This procedure has been automated through the development of an Autopumpdown program that intelligently coordinates the necessary steps across the boundaries of the existing cryogenic and accelerator control systems. This program has greatly reduced both the time and effort required to recover from failures and restart the system. The paper describes the requirements addressed by the program, the steps it takes while operating, the historical development of the program, the spin-off benefits, and the course of anticipated future developments.
\end{abstract}

\section{INTRODUCTION}

The Central Helium Liquefier (CHL) provides refrigeration at three temperature levels for the operation of CEBAF's electron beam linear accelerator (linac) and experimental physics halls. $12 \mathrm{~kW}$ of refrigeration at $38 \mathrm{~K}$ is provided for shield cooling in the $42 \frac{1 / 4}{4}$ cryomodules that make up the accelerator, $10 \mathrm{~g} / \mathrm{sec}$ of $4.5 \mathrm{~K}$ liquid is supplied to the experimental halls to cool superconducting magnets and cryogenic beam targets, and 4.8 $\mathrm{kW}$ of refrigeration at $2 \mathrm{~K}$ is supplied to cool the superconducting radio frequency (RF) cavities inside the cryomodules. The cryomodules are arranged as parallel loads on the refrigerator, each with its own independent Joule-Thompson (JT) inlet valve. When operating, each cryomodule holds approximately $1440 \mathrm{~L}$ of liquid helium.

\footnotetext{
* Supported by U.S. DOE contract DE-AC05-84ER40150
}

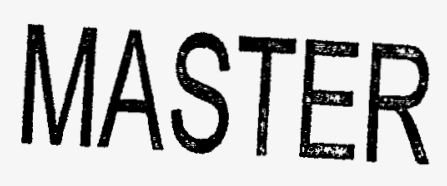


This report has been reproduced from the best available copy.

Available to DOE and DOE contractors from the Office of Scientific and Technical Information, P.O. Box 62, Oak Ridge, TN 37831; prices available from (615)576-8401, FTS 626-8401.

Available to the public from the National Technical Information Service, U.S. Department of Commerce, 5285 Port Royal Rd., Springfield, VA 22161.

Price: $\begin{gathered}\text { Printed Copy } \\ \text { Microfiche } 101\end{gathered}$ 
The CHL is divided into two main sections that were fabricated separately, as shown in Figure 1. The shield cooling and liquefaction loads are supplied by a conventional $4 \mathrm{~K}$ cold box which also feeds the more specialized $2 \mathrm{~K}$ cold box. Operation at $2 \mathrm{~K}$ requires the cryomodule liquid baths to be maintained at an absolute pressure of $0.031 \mathrm{~atm}$. This pressure is achieved by four stages of centrifugal cold compressors (CCs) in the $2 \mathrm{~K}$ cold box. Each compressor stage has its own magnetic bearings and $\mathrm{LN}_{2}$ cooled variable speed electric motor.

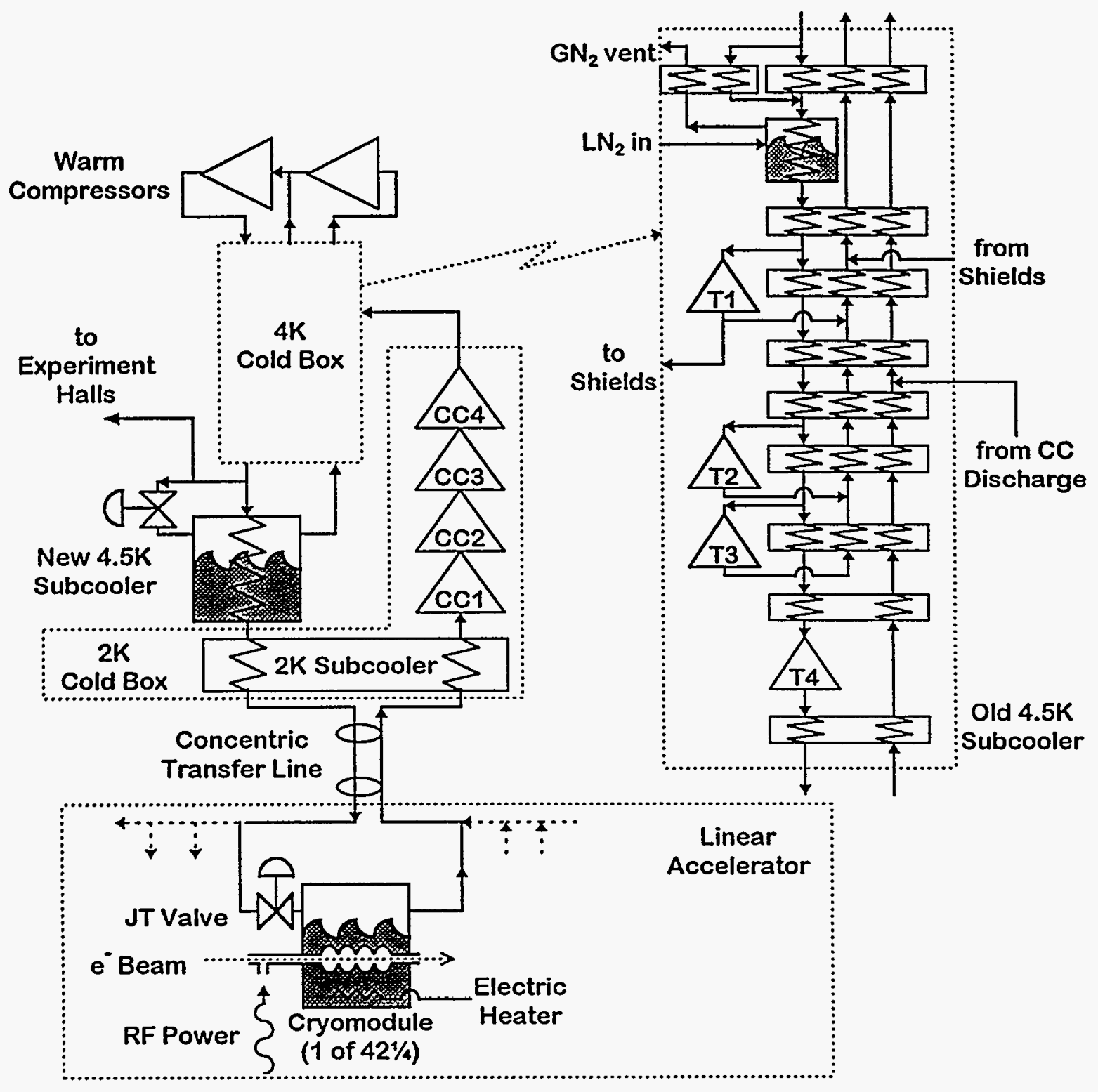

Figure 1. Partial flow diagram for the CEBAF Central Helium Liquefier with an expanded view of the $4 \mathrm{~K}$ cold box. The new $4.5 \mathrm{~K}$ subcooler was installed in the piping between the $4 \mathrm{~K}$ cold box and the $2 \mathrm{~K}$ cold box. For clarity, not all system components are shown.

The pumpdown procedure is the transition from $4 \mathrm{~K}$ operation at $\mathrm{l}$ atm to $2 \mathrm{~K}$ operation at $0.031 \mathrm{~atm}$. This requires coordinating the speeds of the four cold compressors with a variety of other system adjustments. The main goal of the pumpdown is to minimize the time required to prepare the cryomodules for accelerator operations. This involves both 
reaching $2 \mathrm{~K}$ operating pressure and satisfying the minimum liquid levels required in the cryomodules, since the liquid levels drop during pumpdown. This must be accomplished within the constraints of the $4 \mathrm{~K}$ system capacity and the cold compressor surge limits. The centrifugal cold compressors require a minimum inlet volume flow to develop the required pressure ratios without surging. Early in the pumpdown, at high suction pressures, higher mass flow rates are required to satisfy this volume flow requirement. This unloads the $4 \mathrm{~K}$ system, because the $4 \mathrm{~K}$ cold box is running in an unbalanced mode, recovering more gas from the cryomodules than it is supplying as liquid. As the pumpdown progresses, the load on the $4 \mathrm{~K}$ system increases to a maximum (when the return flow through the cold compressors decreases and the liquefaction load to refill the cryomodules is high) and then settles into balanced operation near the completion of the pumpdown.

The CHL Autopumpdown program began as an effort to make the existing $2 \mathrm{~K}$ pumpdown procedure less labor intensive. The manual procedure was the result of months of intensive development by the CEBAF cryogenics staff, often working around the clock to accomplish as much testing as possible during the limited time windows allowed by accelerator commissioning efforts. This initial manual procedure required at least three operator's in two different locations coordinating by telephone. One operator was responsible for the operation of the $4 \mathrm{~K}$ cold box and warm compressors, one for the $2 \mathrm{~K}$ cold box, and one for the heaters and JT valves associated with each of the cryomodules. Progress was often slowed by the difficulty in communicating every new setting between shifts and the resulting non-uniformity of different pumpdown attempts. The Autopumpdown program helped to resolve this problem and free the operators from tedious repetitive tasks to concentrate on refining the procedure. Once it was first implemented, the Autopumpdown software continued to evolve hand in hand with the manual procedure. New procedures were tested manually and those that proved useful were incorporated into the software as quickly as possible, usually the same day.

During the development of the pumpdown procedure, CEBAF was in the midst of changing control systems from TACL (Thaumaturgic Automated Control Logic) to EPICS (Experimental Physics and Industrial Control System). Today the cryogenic systems are still entirely in TACL while the accelerator systems are almost completely in EPICS. Bridging the gap between these systems as they evolved over time was another challenge for the Autopumpdown software. Continuing efforts to convert the cryogenic controls to EPICS are described in Reference 1.

\section{HISTORY}

The first phase of what became the Autopumpdown program was a set of scripts to control the JT valves or heaters in all of the cryomodules simultaneously. These scripts reduced many frantic minutes of typing to a few mouse clicks and confirmations. As the pumpdown procedure firmed up, it became obvious that these operations would be required at certain repeatable pressures at the suction of the cold compressors. As a result, CC suction pressure was chosen as the sequencing variable, and these operations were automated with a preliminary set of independent programs that adjusted the heaters and JT valves at preset pressures as the linacs were pumped down. This automation proceeded in phases. First the heaters were controlled, then the JT valves, and then the cold compressor mass flow setpoint as well.

As the pumpdown procedure continued to become better defined, these simple programs were combined into a master Autopumpdown program that coordinated all of the changes and allowed for other operator actions to be synchronized with them. Cold compressor suction pressure has remained the primary sequencing variable, but individual actions can have their own predefined triggers. For example, as described in the next section, the $30 \mathrm{~K}$ turbine (T2) is jumped through its critical speed range only when the $4 \mathrm{~K}$ 
cold box has warmed sufficiently due to the increasing load to make it necessary, but is prohibited from doing so until the $\mathrm{CC}$ suction pressure is below a given value.

Because the Autopumpdown program evolved along with a continuously changing procedure, very few of its control parameters are hard coded. Most of them are accessible through TACL display pages that are available in both the CHL control room and the accelerator Machine Control Center. This allows for integrated operation of the Autopumpdown with the existing cryogenic controls software and allows the Autopumpdown parameters to be saved and restored along with the rest of the machine control parameters.

\section{THE CURRENT AUTOPUMPDOWN}

Currently the Autopumpdown program performs the following actions, most of which can be controlled from interactive display pages:

- Cold Compressor \#4 Speed: The speed of $\mathrm{CC} 4$ is controlled by a standard PID (Proportional-Integral-Derivative) control loop to maintain a specified mass flow through the $\mathrm{CC}$ train. It is this mass flow (minus the heat load applied in the cryomodules) that determines the rate of pumpdown. However, the flow must be capped due to the limits imposed by the $4 \mathrm{~K}$ plant. The effects of undersized heat exchangers in the $4 \mathrm{~K}$ cold box and undersized warm gas compressors are discussed in Reference 2. The Autopumpdown controls this flow setpoint (and hence CC4 speed) as a function of $\mathrm{CC}$ suction pressure.

- Cold Compressor Speed Ratios: The speeds of CC's 1-3 are controlled as ratios to the speed of CC4. These ratios are determined by theoretical performance curves supplied by the manufacturer and verified to some extent in bench testing, though the scale of the machines limited the bench test capability. Due to stringent accelerator commissioning schedules, there has not yet been sufficient time to re-verify performance data over the full operating ranges of the machines. Operating experience, however, has indicated that there are substantial deviations from the theoretical predictions. The Autopumpdown program accounts for this deviation by allowing the ratios to be controlled on the theoretical curves only for specific "safe" intervals. In the earliest phase of the pumpdown the ratios are held fixed, during the intermediate phases they are adjusted with offsets from the theoretical curves, and in the last phase they are limited to experimentally determined values. See also Final Adjustments below.

- JT Valve Positions: The JT valve positions for each of the cryomodules are controlled by separate PID loops to maintain liquid level in each module. The Autopumpdown adjusts cryomodule liquid level setpoints (and hence JT valve-positions) as a function of CC suction pressure. This is necessary both to provide enough flow to start the pumpdown and to slightly overfill the cryomodules so that the final liquid level after mass has been removed by the pumpdown is within range for accelerator RF operations to begin as soon as possible.

- Cryomodule Heaters: Each cryomodule contains a string of four heater elements that are used for load-leveling purposes. Software within the RF control system monitors the calculated heat load deposited in each cryomodule by the RF power and adjusts these heaters to keep the total load per module at a requested value. The Autopumpdown program communicates with the RF control software to adjust the requested heat in each cryomodule as a function of $\mathrm{CC}$ suction pressure. This feature was used extensively in early pumpdowns to slow the rate of pumpdown in order to analyze the problems encountered at particular pressure ranges. Currently it is used 
exclusively to perform a "soft landing" at the end of the pumpdown, when heat is applied gradually to halt the pumpdown at a specific target pressure.

- 4.5K Subcooler: One of the crucial breakthroughs of the development phase of the pumpdown procedure was the determination that the cold compressors were much more susceptible to fluctuations in inlet temperature during the early stages of a pumpdown than during the later stages. The inlet temperature to the cold compressor train is closely coupled through a $2 \mathrm{~K}$ subcooler heat exchanger to the temperature of the helium being supplied to the load. This temperature in turn is controlled by conditions within a special $4.5 \mathrm{~K}$ boiler/subcooler installed by CEBAF when it was found that the originally supplied 4.5K subcooler was inadequate. (See Reference 2.) Keeping this subcooler at low liquid levels and high pressures during the early part of a pumpdown (in order to elevate the CC suction temperature) greatly enhances the stability of the cold compressors. The boiler must eventually be filled, however, or the $\mathrm{CC}$ exhaust becomes too hot for the $4 \mathrm{~K}$ cold box and the supply temperature to the load becomes too high. The Autopumpdown program makes this transition gradually as a function of $\mathrm{CC}$ suction pressure when the cold compressors are operating in a range less sensitive to inlet temperature.

- Turbine Speeds: The 4K capacity required to support a pumpdown varies significantly over the course of the pumpdown. Capacity is normally controlled by PID loops that regulate the inlet valves to the turbines in the $4 \mathrm{~K}$ cold box. However, due to the fixed brake pressures and critical resonant frequencies of the turbines, large portions of their speed ranges are forbidden. This results in each turbine (except the coldest) having two operating modes, above and below its critical speed range. Critical speeds are avoided by limits placed on inlet valve motion. When it is necessary to cross the critical speed ranges, rapid changes are made to inlet valve position to quickly "jump" the rotor speed up or down. If the rotor spends too long in the prohibited range, it is shut down by the local turbine controller. Because the critical speed ranges are so wide, a turbine that is "jumped" up in speed because its outlet temperature is too high may immediately trip itself off on low outlet temperature. The Autopumpdown manages the speeds of the second and third turbines to meet the capacity requirements of the pumpdown within the operating constraints of the turbines themselves. This includes controlling the "jumps" through critical speeds.

- RF Power Interlocks: During a pumpdown, operation of RF power in the cryomodules is prohibited in order to stabilize the heat load. The Autopumpdown notifies the RF control system through a set of software interlocks when $R F$ is prohibited. Once the target CC suction pressure is reached, the Autopumpdown notifies the RF control system that RF power is again allowed.

- Controlling Pressure Device: A chronic problem during cold compressor testing was the failure of various pressure transducers in the $2 \mathrm{~K}$ cold box. As a result, the Autopumpdown allows the selection of controlling pressure transducer from a list of devices tied to various points on the CC suction path. Optionally, it will automatically switch from the selected high range device to the default low range device at a specified pressure.

- Final Adjustments: It has been found that the cold compressors operate more stably and robustly at steady state when they have more nearly equal pressure ratios across the stages than those determined by the theoretical performance curves. Accordingly, once the target pressure has been reached, the Autopumpdown gradually adjusts the CC speed ratios to specified values to equalize the pressure ratios across the four stages. 
Once the speed ratio adjustments are complete, the cold compressor flow is reduced to a lower value made possible by the more stable operation.

\section{BEYOND AUTOPUMPDOWN}

Besides the Autopumpdown itself, a small suite of additional control programs has grown out of the techniques developed. The net result is a dramatic decrease in both the human intervention required to operate the $\mathrm{CHL}$ and the time to recovery in the event of a cold compressor trip.

\section{Auto Heater Control}

The linac pressure is regulated during $2 \mathrm{~K}$ operation by a $990 \mathrm{~W}$ "swing" heater made up of the heaters contained in six of the cryomodules. This swing heater is under PID regulation from the main cryogenic control software. The Automatic Heater Control program continuously monitors the "swing" heater performance and augments it by adjusting the heaters in the remaining 36 cryomodules that are available for control. These remaining heaters are controlled by the accelerator EPICS control system and are not directly accessible to the cryogenics TACL control system, except through the Auto Heater Control program. These are the same heaters that are adjusted by the Autopumpdown program and they are accessed in the same way. This effectively extends the useful range of the swing heater from $990 \mathrm{~W}$ to over $4 \mathrm{~kW}$ to compensate for changes in heat load, such as a sudden loss of RF power in the accelerator. This capability has prevented many shutdowns of the $2 \mathrm{~K}$ system, especially during the early phases of the EPICS conversion when RF control software was unreliable.

The Auto Heater Control program also has the responsibility of shutting off all heat and RF power in the accelerator in the event of a cold compressor shutdown. When the cold compressors trip, the linac begins to pressurize and any heat load on the cryomodules tends to warm and expand the $2 \mathrm{~K}$ liquid. Since accelerator operation requires the cryomodules to be $90 \%$ full at all times, expanding liquid will overflow into the return transfer line. Overflowing liquid pools in the return line where it reduces the flow area and boils off, causing flow instabilities. When there has been substantial overflowing into the return line, the time required for a pumpdown increases dramatically and the probability of success for an immediate pumpdown is greatly reduced. Both of these effects are mitigated by automatic shutoff of all heater and RF loads immediately after a trip.

\section{Autobackfill}

The Autobackfill program was designed to automate the tasks required to prepare the system to be pumped down again after a trip. It is not initiated automatically to avoid losing any information that might be useful in diagnosing the cause of the trip. An operator must launch the program after investigating (and correcting if necessary) the cause of the cold compressor trip. When the cold compressors shut down for any reason, isolation valves on the $2 \mathrm{~K}$ cold box close automatically. In order to protect the cold compressors, the programmable logic controller (PLC) on the $2 \mathrm{~K}$ cold box will not allow these valves to be opened or the CC motors to be started until the cryomodules have been re-pressurized through bypass valves.

The Autobackfill program will reset all latched faults on the cold compressor controllers, open bypass valves to pressurize the cryomodules and return transfer line, open cooldown valves to bring the cold compressor suction temperature into the acceptable range for a pumpdown, open the isolation valves around the $2 \mathrm{~K}$ cold box, and start the cold compressor motors. While backfilling and cooling down, the program monitors for and avoids undesirable conditions of bringing the main compressor suction header 
subatmospheric and producing liquid in the cold compressor suction header. When all parameters are in range, the program closes bypass and cooldown valves and exits. The machine is then ready to pump down. In the event of a cold compressor trip due to some transient condition that doesn't damage any hardware, the system can be restored to full $2 \mathrm{~K}$ operation by simply activating the Autobackfill followed immediately by the Autopumpdown.

\section{Autoramp}

This simple utility program allows any operator input parameter in the cryogenic system to be smoothly and automatically ramped to a target value at a fixed rate. The operator invokes the program and then specifies the name of the signal to be controlled, the target value, and the ramp rate. Once the input is confirmed, the ramp is carried out and the program exits.

\section{FUTURE DIRECTIONS}

The long evolutionary process has resulted in an Autopumpdown program carefully tailored to perform all the complex operations of a cold compressor pumpdown. More than $90 \%$ of the time no operator intervention is required once the Autopumpdown has been initiated, and very little intervention is usually required in the remaining runs. Effort is still underway to improve this hands-off success rate. After each pumpdown where operators are required to intercede, the reasons for the intervention are analyzed. If the causes are sufficiently likely to occur again, a standard response is developed and integrated into the Autopumpdown code.

As the list of control parameters that have been determined to have a direct impact on the cold compressor pumpdown process has grown, more and more signals have come under the control of the Autopumpdown. Many of these signals, however, do not require repeated updates during the pumpdown and need only be set once on startup. Currently this process is still largely manual; an operator compares values from the computer against a standard set in hardcopy, looking for discrepancies. A future extension of the Autopumpdown program will keep these settings in human-readable files, separate from the bulk of control system saved settings. The program will then be able to ensure that all necessary startup values are correct.

More radical modifications to the pumpdown procedure are under consideration for testing when the accelerator operation schedule permits. The most interesting of these options concern manipulations in the speed ratios of the various cold compressor stages.

\section{ACKNOWLEDGMENTS}

Development of the cold compressor pumpdown procedures was a group effort involving the entire CEBAF Cryogenics staff (D. Arenius, V. Ganni, D. Kashy, T. Reid, J. Wilson, and the authors) with the assistance and direction of C. Rode. D. Wetherholt of the CEBAF Instrumentation and Controls group developed a real-time plotting program to monitor the cold compressors in dimensionless phase space that was invaluable during development of the pumpdown procedures.

\section{REFERENCES}

1. M. S. Keesee, et al. "The CEBAF Control System for the CHL," to be published in the 1995 Advances in Cryogenic Engineering. 
2. W. C. Chronis, et al. "Commissioning of the CHL Refrigerator at CEBAF," to be published in the 1995 Advances in Cryogenic Engineering. 


\section{DISCLAIMER}

This report was prepared as an account of work sponsored by an agency of the United States Government. Neither the United States Government nor any agency thereof, nor any of their employees, makes any warranty, express or implied, or assumes any legal liability or responsibility for the accuracy, completeness, or usefulness of any information, apparatus, product, or process disclosed, or represents that its use would not infringe privately owned rights. Reference herein to any specific commercial product, process, or service by trade name, trademark, manufacturer, or otherwise does not necessarily constitute or imply its endorsement, recommendation, or favoring by the United States Government or any agency thereof. The views and opinions of authors expressed herein do not necessarily state or reflect those of the United States Government or any agency thereof. 


\section{DISCLAIMER}

Portions of this document may be illegible in electronic image products. Images are produced from the best available original document. 